\title{
A photometric study of NN Virginis $\star$
}

\author{
G. Djurašević ${ }^{1}$, B. Albayrak ${ }^{2}$, T. Tanrıverdi ${ }^{2}$, and S. Erkapić ${ }^{1}$ \\ 1 Astronomical Observatory, Volgina 7, 11160 Belgrade, Serbia and Montenegro and Isaac Newton Institute of Chile, \\ Yugoslav branch \\ 2 Ankara University, Faculty of Science, Dept. of Astronomy and Space Sciences, 06100, Tandoğan, Ankara, Turkey \\ Received 25 February 2003 / Accepted 14 October 2003
}

\begin{abstract}
Photoelectric $B V$ light curves of the recently discovered eclipsing binary NN Vir were studied for the first time to derive the physical parameters of the system. The light curves were obtained at the Ankara University Observatory during three nights in May, 2002. The solutions made by using Djurasevic's inverse problem method describe the NN Vir system as a high overcontact configuration $\left(f_{\text {over }} \sim 58 \%\right)$ with a relatively small temperature differences between the components $\left(\Delta T=T_{\mathrm{h}}-T_{\mathrm{c}} \sim 160 \mathrm{~K}\right)$. These solutions suggest a significant mass and energy transfer from the more massive primary onto the less massive secondary. The hot area on the less massive star, near the neck region, can be taken as a consequence of this mass and energy exchange between the components through the connecting neck of the common envelope.
\end{abstract}

Key words. binaries: eclipsing - binaries: close - individual: NN Virginis

\section{Introduction}

NN Vir was discovered to be a variable star by the Hipparcos satellite (ESA 1997). The Hipparcos photometric observations of the system show a light curve variation with an amplitude of 0.41 ranging from 7.605 to 8.015 . According to Woitas (1997), this star was an RR Lyrae type variable with a 0.20 day period. Gomez-Ferrelland \& Garcia-Melendo (1997) correctly classified the system as a W Ursa Majoris (W UMa) type eclipsing binary. They published a light curve obtained in the $V$ band using a 6-cm telescope, and determined the initial light elements of the system. The light curve shows two equal-depth minima with an amplitude of 0.37 . They also published four times of minima of NN Vir. Rucinski \& Lu (1999) obtained radial velocity curves of both components and determined a mass ratio of $q=m_{\mathrm{c}} / m_{\mathrm{h}}=0.491$. The indices (h, c) refer to the hotter more massive and cooler less massive component, respectively.

Rucinski \& Lu pointed out that the spectral type of the system is $F 0 / F 1 \mathrm{~V}$ and that it belongs to the A sub-class of W UMa configuration. We observed and analyzed the system in order to obtain its full modelling.

\section{The observational data and light curves}

The differential $B V$ observations of NN Vir were carried out on 3 nights in May of 2002 at the Ankara University Observatory,

Send offprint requests to: G. Djurašević, e-mail: gdjurasevic@aob.bg.ac.yu

* Table 2 is only available in electronic form at CDS via anonymous ftp to cdsarc.u-strasbg.fr $(130.79 .128 .5)$ or via http://cdsweb.u-strasbg.fr/cgi-bin/qcat?J/A+A/415/283
Table 1. The catalogue information for NN Vir, the comparison and check stars.

\begin{tabular}{lrrr}
\hline \hline Parameter & NN Vir & Comparison & Check \\
\hline BD & $+06^{\circ} 2869$ & $+06^{\circ} 2864$ & $+05^{\circ} 2865$ \\
HD & 125488 & 124970 & \\
$\alpha_{2000}$ & $14^{\mathrm{h}} 19^{\mathrm{m}} 38^{\mathrm{s}}$ & $14^{\mathrm{h}} 16^{\mathrm{m}} 35^{\mathrm{s}}$ & $14^{\mathrm{h}} 18^{\mathrm{m}} 26^{\mathrm{s}}$ \\
$\delta_{2000}$ & $+05^{\circ} 53^{\prime} 47^{\prime \prime}$ & $+05^{\circ} 31^{\prime} 55^{\prime \prime}$ & $+05^{\circ} 13^{\prime} 25^{\prime \prime}$ \\
$V$ & $7^{\mathrm{m}} 64^{a}$ & $8.33^{a}$ & $9^{\mathrm{m}} \cdot 95$ \\
$B-V$ & $0^{\mathrm{m}} 407^{a}$ & $0^{\mathrm{m}} 438^{a}$ & \\
\hline
\end{tabular}

${ }^{a}$ From Hipparcos Catalogue (ESA 1997).

by using a SSP-5A photometer attached to a 30-cm Maksutov telescope. $\mathrm{BD}+06^{\circ} 2864$ and $\mathrm{BD}+05^{\circ} 2865$ were used as comparison and check stars, respectively. A total of 217 observations was secured in each filter. The observations, in the sense variable minus comparison, were corrected for atmospheric extinction using the extinction coefficients obtained for each night from the observations of the comparison star. During the observations no variations in brightness were detected for the comparison and check stars. The probable error of a single observation point was estimated to be \pm 0.020 and \pm 0.026 in $B$ and $V$ colours, respectively.

All differential data were transformed to standard $B, V$ magnitudes using the converting coefficients of the local system determined by Müyesseroğlu (2003). Standard $B V$ indices for NN Vir, the comparison and check stars are given in Table 1 together with the relevant catalogue information for these stars. Table 2 (accessible in electronic form) lists the heliocentric Julian Dates, orbital phases, standard $B$ and $V$ magnitudes of the system. 


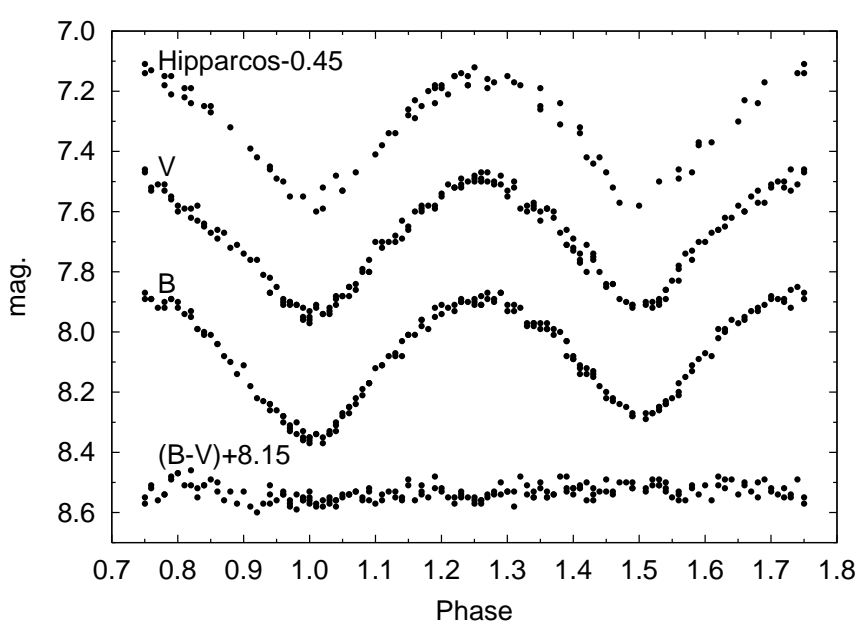

Fig. 1. Hipparcos and new standard $B, V$ light, and $B-V$ colour curves of NN Vir.

Table 3. The light levels with the errors in the estimates and their differences in the light curves of NN Vir.

\begin{tabular}{lccc}
\hline \hline & $B$ & $V$ & Hip \\
\hline Max. light at 0.25 & $7.896 \pm 0.012$ & $7.493 \pm 0.015$ & $7.609 \pm 0.029$ \\
Max. light at 0.75 & $7.887 \pm 0.026$ & $7.502 \pm 0.029$ & $7.581 \pm 0.012$ \\
Min. light at 0.00 & $8.351 \pm 0.013$ & $7.938 \pm 0.022$ & $8.031 \pm 0.024$ \\
Min. light at 0.50 & $8.271 \pm 0.011$ & $7.910 \pm 0.008$ & $8.026 \pm 0.012$ \\
$\Delta \max \left(m_{0.25}-m_{0.75}\right)$ & 0.009 & -0.009 & 0.028 \\
$\Delta \min \left(m_{0.00}-m_{0.50}\right)$ & 0.080 & 0.028 & 0.005 \\
Depth of Min. I & 0.455 & 0.445 & 0.417 \\
Depth of Min. II & 0.384 & 0.408 & 0.445 \\
\hline
\end{tabular}

The light and colour curves formed by the transformed magnitudes and colours are shown in Fig. 1 together with the Hipparcos light curve. The photometric phases of the light and colour curves were calculated with Eq. (1). The light levels estimated by averaging data around the maxima and minima (by taking a $\Delta \phi= \pm 0.02$ interval) and their differences are listed in Table 3 . It can be seen in Fig. 1 and Table 3 that the primary minimum in our light curves are deeper than the secondary minimum in both $B$ and $V$ colours while in the light curve of Hipparcos the depths of the two minima are almost equal in the light curve of Hipparcos. Thus, what Gomez-Forreland \& Garcia-Melendo (1997) and Albayrak et al. (2002) call type I (the primary minimum) should be called type II (the secondary minimum) and vice versa. In our observations, there is no significant difference between the levels of the two maxima in both colours while in the Hipparcos light curve the light at phase 0.75 ( $\max$ II) is brighter than at phase 0.25 ( $\max$ I). The shape of the light curve in Fig. 1 indicates the overcontact configuration of the system. Figure 1 also shows a slight phase dependence of the colour curve $(B-V)$ which is more easily seen in Fig. 2. This can be a consequence of the system activity.

The observations cover two minima. We also obtained three minima for NN Vir in 2002 during the observational program of selected eclipsing binaries. Their timings were calculated using the method of Kwee \& van Woerden (1956), and they have already been published by Albayrak et al. (2002). The times of minimum of NN Vir together with four more photoelectric
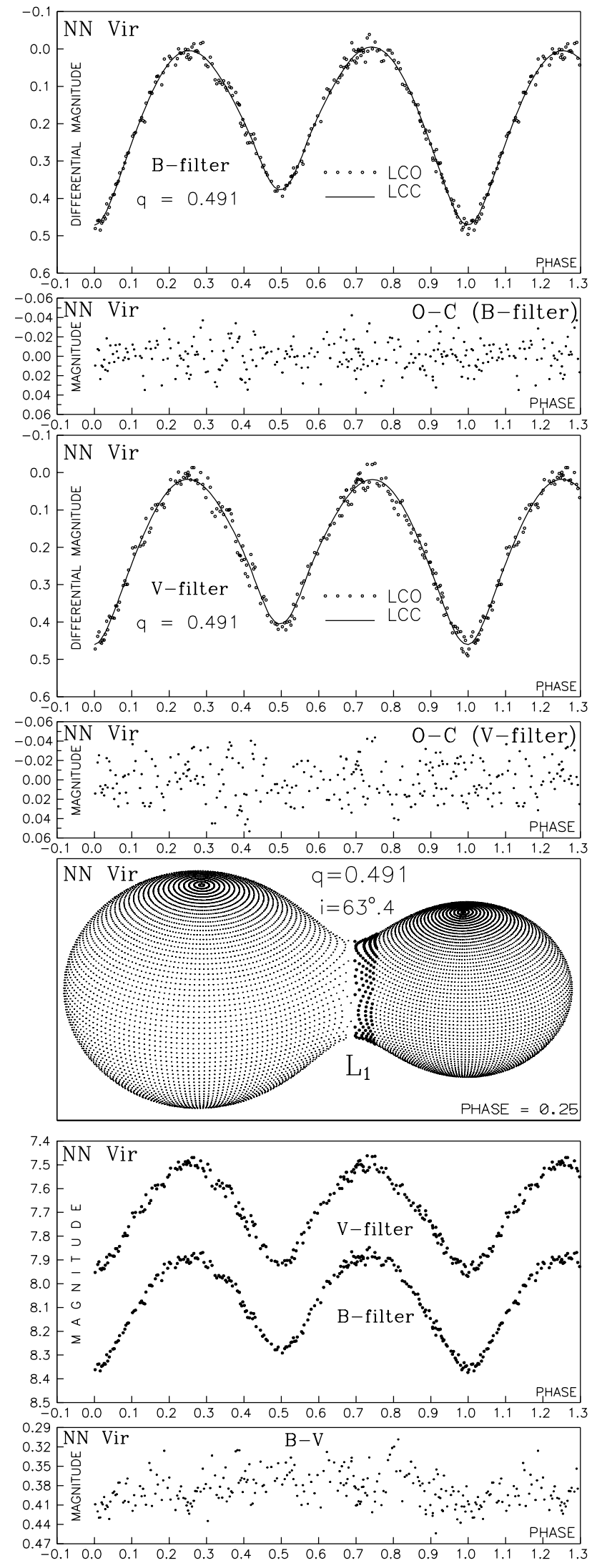

Fig. 2. Observed (LCO) and final synthetic (LCC) light curves of the NN Vir with final O-C residuals obtained by analysing the $B$ and $V$ observations, the view of the system at orbital phase 0.25 , obtained with parameters estimated from the observations, and $B, V$, and $B-V$ colour curves. 
minima published by Gomez-Forrelland \& Garcia-Melendo (1997) yielded the following linear light elements:

HJD MinI $=2450520.5962(5)+0.48068667(2) \times E$.

\section{The light curve analysis}

To estimate the parameters of NN Vir, we used Djurašević's (1992a) programme generalised to the case of an overcontact configuration (Djurašević et al. 1998). The programme is based on the Roche model and the principles arising from the paper by Wilson \& Devinney (1971). The light-curve analysis was made by applying the inverse-problem method (Djurašević 1992b) based on Marquardt's (1963) algorithm.

According to this method, the stellar size in the model is described by the filling factors for the critical Roche lobes $F_{\mathrm{h}, \mathrm{c}}$ of the primary and secondary component, respectively, which tell us to what degree the stars in the system fill their corresponding critical lobes. For synchronous rotation of the components, these factors are expressed as the ratio of the stellar polar radii, $R_{\mathrm{h}, \mathrm{c}}$, and the corresponding polar radii of the critical Roche lobes, i.e., $F_{\mathrm{h}, \mathrm{c}}=R_{\mathrm{h}, \mathrm{c}} / R_{\text {Roche }_{\mathrm{h}, \mathrm{c}}}$. In the case of an overcontact configuration the potential $\Omega_{\mathrm{h}, \mathrm{c}}$ characterising the common photosphere, is derived with a filling factor of the critical Roche lobe $F_{\mathrm{h}}>1$ of the primary, while the factor $F_{\mathrm{c}}$ may be excluded from further consideration. The degree of overcontact is defined in the classical way (Lucy \& Wilson 1979) as:

$f_{\text {over }}[\%]=100 \cdot\left(\Omega_{\mathrm{h}, \mathrm{c}}-\Omega_{\mathrm{i}}\right) /\left(\Omega_{\mathrm{o}}-\Omega_{\mathrm{i}}\right)$,

where $\Omega_{\mathrm{h}, \mathrm{c}}, \Omega_{\mathrm{i}}$, and $\Omega_{\mathrm{o}}$ are the potentials of the common photosphere and of the inner and outer contact surfaces, respectively.

The present analysis yields $F_{\mathrm{h}}>1$ for the filling coefficient in the critical Roche lobe, i.e., the overcontact configuration. Tidal effects are expected to contribute to synchronisation of the rotational and orbital periods. Therefore, in the inverse problem we adopted $f_{\mathrm{h}, \mathrm{c}}=\omega_{\mathrm{h}, \mathrm{c}} / \omega_{\mathrm{K}}=1.0$ for nonsynchronous rotation coefficients, where $f_{\mathrm{h}, \mathrm{c}}$ is the ratio of the angular rotation rate $\left(\omega_{\mathrm{h}, \mathrm{c}}\right)$ to the Keplerian $\left(\omega_{\mathrm{K}}\right)$ orbital revolution rate.

In the analysis of the light curves, instead of the often used and somewhat questionable practice of forming normal points, we used the original observational data in order to avoid negative influences of such normalization. The mass ratio of the components was fixed in the inverse problem at $q=m_{\mathrm{c}} / m_{\mathrm{h}}=$ 0.491, estimated by Rucinski \& Lu (1999) from radial velocity solution. Based on the spectral type of F0/F1 V the temperature of the more massive component, in agreement with Popper's (1980) calibration, was set at $T_{\mathrm{h}}=6900 \mathrm{~K}$. Of course, the temperature distribution over the stellar surface depends on the gravity-darkening and reflection effects.

To achieve more reliable estimates of the model parameters in the light-curve analysis programme, we applied a quite dense coordinate grid, having $72 \times 144=10368$ elementary cells per star. The intensity and angular distribution of radiation of elementary cells are determined by the stellar effective temperature, limb-darkening, gravity-darkening and by the effect of reflection in the system.

For the gravity-darkening exponents of the stars we used the theoretical predictions obtained on the basis of
Claret's (1998) tables. On the basis of the masses of the components, by interpolation in the stellar effective temperature $T_{\text {eff }}$ and surface gravity $\log g$ from Claret's (1998) tables we have the values of the gravity-darkening exponents $\beta_{\mathrm{h}}=0.06$ and $\beta_{\mathrm{c}}=0.12$, appropriate for stars with convective envelopes. Consequently, their albedos, $A_{\mathrm{h}, \mathrm{c}}$, were set at 0.5 .

A non-linear limb-darkening law has been used in the present work to avoid the possible negative influence of the wrong evaluation of limb-darkening coefficients on other parameters in the inverse problem. In agreement with Claret's (2000) paper, whose tables we used, we chose the new approximation which can be written as:

$$
\begin{aligned}
\frac{I(\mu)}{I(1)}= & 1-a_{1}\left(1-\mu^{1 / 2}\right)-a_{2}(1-\mu)-a_{3}\left(1-\mu^{3 / 2}\right) \\
& -a_{4}\left(1-\mu^{2}\right)
\end{aligned}
$$

where $a_{1,2,3,4}$ are the limb-darkening passband specific coefficients and $\mu=\cos \gamma . I(1)$ is the passband specific intensity at the center of the stellar disc, and $\gamma$ is the angle between the line of sight and the emergent flux. This law, based on the Least-Squares Method, is able to describe the intensity distribution over the whole stellar disk very well, with very good flux conservation. For a given metallicity, the values of the passband limb-darkening coefficients are derived from the current values of the stellar effective temperature $T_{\text {eff }}$ and surface gravity $\log g$ in each iteration, by interpolation for both of these quantities in Claret's (2000) tables. This was achieved by bi-linear interpolation (Press et al. 1992).

The computer programme for the light curve analysis can be run choosing among three possibilities for the treatment of the radiation law: 1) simple black-body theory, 2) stellar atmosphere models by Carbon \& Gingerich (1969) (CG), and 3) the Basel Stellar Library (BaSeL).

The present light-curve analysis was carried out using all three possibilities. The final choice of the approximation used in the light-curve analysis is made in such a way as to keep the disagreement between different individual passbands solutions at a minimum. In this case we found that BaSeL model flux distributions, with the assumed solar chemical abundance for the components of the system $([\mathrm{Fe} / \mathrm{H}]=0)$, provided better agreement between the individual $B$ and $V$ solutions than the simple black-body theory or CG stellar atmosphere models.

We have explored the "corrected" BaSeL model flux distributions, consistent with extent empirical calibrations (Lejeune et al. 1997, 1998). In solving the inverse problem of the lightcurve analysis, the fluxes are calculated in each iteration for current values of temperatures and $\log g$, by bi-linear interpolation (Press et al. 1992) for both of these quantities in the atmosphere tables for a given metallicity $[\mathrm{Fe} / \mathrm{H}]$ of the components. The surface gravities can be derived very accurately from the masses and radii of CB stars, but the temperature determination is related to the assumed metallicity and strongly depends on photometric calibration.

For a successful application of this model in the analysis of the observed light curves, the inverse-problem method proposed by Djurašević (1992b) was used. Optimum model parameters are obtained through the minimization of $\Sigma(\mathrm{O}-\mathrm{C})^{2}$, where $\mathrm{O}-\mathrm{C}$ is the residual between the observed (LCO) and 
synthetic (LCC) light curves for a given orbital phase. The minimisation of $\Sigma(\mathrm{O}-\mathrm{C})^{2}$ is done in an iterative cycle of corrections of the model parameters by using the modified Marquardt (1963) algorithm. In this way, the inverse-problem method provides estimates of the system parameters and their standard errors.

The light curves of NN Vir have a peculiar shape. The analysis has shown that this system has a very distinct overcontact configuration where during the deeper (primary) minimum the less massive and smaller component partially eclipses the more massive and larger one. However, such a simplified model is not sufficient to describe all the features seen in the observed light curves. We have tested several different hypotheses in the attempt to provide a good fit of the observations by varying the albedos, the gravity-darkening exponents of the components of the system and the degree of overcontact. It turned out that we could not obtain a combination giving us a satisfactory solution. However, these attempts to treat the albedos of the components as free parameters of the inverse problem have shown that the obtained solutions demanded that the albedo of the secondary (cooler) component has to be much larger than the expected value. This suggests that on the secondary opposite the Lagrangean point $L_{1}$, near the neck region, we have an area with increased temperature. So, we fixed the albedos and gravity darkening exponents to their theoretically expected values, and introduced a hot region as a possible natural explanation for the behaviour of the light curve. The hot region can be explained as a consequence of an intensive mass and energy exchange between the components through the neck connecting the common envelope, i.e., of a mechanism leading to almost equal stellar temperatures. Finally, in the hypothesis of a hot region in the neck zone of the secondary we have a solution that seems logical and provides a relatively good fit of the observations, with mutually consistent solutions from the analysis of individual $B$ and $V$ light curves.

In our code this active hot region on the secondary component is approximated by a circular spot, characterised by the temperature contrast of the spot with respect to the surrounding photosphere $\left(A_{\mathrm{S}}=T_{\mathrm{S}} / T_{\mathrm{c}}\right)$, by the angular dimension (radius) of the spot $\left(\theta_{\mathrm{S}}\right)$ and by the longitude $\left(\lambda_{\mathrm{S}}\right)$ and latitude $\left(\varphi_{\mathrm{S}}\right)$ of the spot centre. The longitude $\left(\lambda_{\mathrm{S}}\right)$ is measured clockwise (as viewed from the direction of the $+Z$-axis) from the $+X$-axis (line connecting the star centers) in the range $0^{\circ}-360^{\circ}$. The latitude $\left(\varphi_{\mathrm{S}}\right)$ is measured from $0^{\circ}$ at the stellar equator (orbital plane) to $+90^{\circ}$ towards the "north" $(+Z)$ and $-90^{\circ}$ towards the "south" $(-Z)$ pole. The assumed physical nature of the hot region allowed an approximation on its location, which also reduced the number of free model parameters: it is in the equatorial zone of the secondary $\left(\varphi_{S}=0^{\circ}\right)$.

\section{Results and discussion}

Within the working hypothesis we chose the inverse problem of the optimisation of system parameters provides good mutual consistency between the solutions obtained in the analyses of individual $B$ and $V$ light curves. These solutions are given in Table 4 . The first three rows of the table present the number
Table 4. Results of the analysis of NN Vir light curves obtained by solving the inverse problem for the Roche model including the active hot region on the cooler secondary component.

\begin{tabular}{|c|c|c|}
\hline Quantity & $B$-filter & $V$-filter \\
\hline$n$ & 217 & 217 \\
\hline$\Sigma(\mathrm{O}-\mathrm{C})^{2}$ & 0.0500 & 0.0890 \\
\hline$\sigma$ & 0.0152 & 0.0202 \\
\hline$q=m_{\mathrm{c}} / m_{\mathrm{h}}$ & 0.491 & \\
\hline$T_{\mathrm{h}}$ & 6900 & \\
\hline$\beta_{\mathrm{h}}$ & 0.06 & \\
\hline$\beta_{\mathrm{c}}$ & 0.12 & \\
\hline$A_{\mathrm{h}}=A_{\mathrm{c}}$ & 0.5 & \\
\hline$f_{\mathrm{h}}=f_{\mathrm{c}}$ & 1.0 & \\
\hline$T_{\mathrm{c}}$ & $6676 \pm 43$ & $6796 \pm 66$ \\
\hline$F_{\mathrm{h}}$ & $1.073 \pm 0.001$ & $1.075 \pm 0.001$ \\
\hline$i\left[^{\circ}\right]$ & $63.3 \pm 0.2$ & $63.4 \pm 0.2$ \\
\hline$A_{\mathrm{S}}=T_{\mathrm{S}} / T_{\mathrm{c}}$ & $1.22 \pm 0.01$ & $1.25 \pm 0.02$ \\
\hline$\theta_{\mathrm{S}}\left[^{\circ}\right]$ & $34.1 \pm 0.6$ & $32.2 \pm 1.0$ \\
\hline$\lambda_{\mathrm{S}}\left[^{\circ}\right]$ & $182.2 \pm 0.4$ & $179.6 \pm 0.7$ \\
\hline$\varphi_{\mathrm{S}}\left[^{\circ}\right]$ & 0.0 & 0.0 \\
\hline$a_{1}^{\mathrm{h}, \mathrm{c}}$ & $+0.3071,+0.3051$ & $+0.3738,+0.3739$ \\
\hline$a_{2}^{1, c}$ & $+0.9694,+0.8873$ & $+0.8779,+0.8602$ \\
\hline$a_{3}^{\mathrm{h}, \mathrm{c}}$ & $-0.6385,-0.4883$ & $-0.7808,-0.7433$ \\
\hline$a_{4}^{\mathrm{h}, \mathrm{c}}$ & $+0.1788,+0.1195$ & $+0.2633,+0.2468$ \\
\hline$\Omega_{\mathrm{h}, \mathrm{c}}$ & 2.6906 & 2.6856 \\
\hline$\Omega_{\mathrm{in}}$ & 2.8585 & 2.8585 \\
\hline$\Omega_{\text {out }}$ & 2.5647 & 2.5647 \\
\hline$f_{\text {over }}[\%]$ & 57.15 & 58.86 \\
\hline$R_{\mathrm{h}}[D=1]$ & 0.446 & 0.447 \\
\hline$R_{\mathrm{c}}[D=1]$ & 0.330 & 0.331 \\
\hline$L_{\mathrm{h}} /\left(L_{\mathrm{h}}+L_{\mathrm{c}}\right)$ & 0.657 & 0.636 \\
\hline $\mathcal{M}_{\mathrm{h}}\left[M_{\odot}\right]$ & $1.34 \pm 0.02$ & \\
\hline $\mathcal{M}_{\mathrm{c}}\left[M_{\odot}\right]$ & $0.66 \pm 0.02$ & \\
\hline $\mathcal{R}_{\mathrm{h}}\left[R_{\odot}\right]$ & $1.58 \pm 0.02$ & \\
\hline $\mathcal{R}_{\mathrm{c}}\left[R_{\odot}\right]$ & $1.19 \pm 0.02$ & \\
\hline $\log g_{\mathrm{h}}$ & $4.17 \pm 0.02$ & \\
\hline $\log g_{\mathrm{c}}$ & $4.11 \pm 0.02$ & \\
\hline$M_{\mathrm{bol}}^{\mathrm{h}}$ & $3.02 \pm 0.03$ & \\
\hline$M_{\mathrm{bol}}^{\mathrm{c}}$ & $3.74 \pm 0.05$ & \\
\hline$a_{\mathrm{orb}}\left[R_{\odot}\right]$ & $3.248 \pm 0.009$ & \\
\hline
\end{tabular}

Note: $n$ - number of observations, $\Sigma(\mathrm{O}-\mathrm{C})^{2}-$ final sum of squares of residuals between observed and synthetic light curves, $\sigma-$ standard deviation of the observations, $q=m_{\mathrm{c}} / m_{\mathrm{h}}$ - mass ratio of the components, $T_{\mathrm{h}, \mathrm{c}}$ - temperature of the hotter primary and cooler secondary, $\beta_{\mathrm{h}, \mathrm{c}}, A_{\mathrm{h}, \mathrm{c}}, f_{\mathrm{h}, \mathrm{c}}-$ gravity-darkening exponents, albedos and nonsynchronous rotation coefficients of the components respectively, $F_{\mathrm{h}}$ - filling factor for the critical Roche lobe of the hotter primary, $i\left[^{\circ}\right]$ - orbit inclination (in arc degrees), $A_{\mathrm{S}}$ - hot-spot temperature factor, $\theta_{\mathrm{S}}, \lambda_{\mathrm{S}}, \varphi_{\mathrm{S}}-$ hot-spot radius, longitude and latitude (in arc degrees), $a_{1}^{\mathrm{h}, \mathrm{c}}, a_{2}^{\mathrm{h}, \mathrm{c}}, a_{3}^{\mathrm{h}, \mathrm{c}}, a_{4}^{\mathrm{h}, \mathrm{c}}-$ nonlinear limb-darkening coefficients of the components (Claret's formula), $\Omega_{\mathrm{h}, \mathrm{c}}, \Omega_{\mathrm{in}}, \Omega_{\mathrm{out}}$-dimensionless surface potentials of the components and of the inner and outer contact surfaces respectively, $f_{\text {over }}[\%]$ - degree of overcontact, $R_{\mathrm{h}, \mathrm{c}}-$ polar radii of the components in units of the distance between the component centres, $L_{\mathrm{h}} /\left(L_{\mathrm{h}}+L_{\mathrm{c}}\right)$ - luminosity of the more massive hotter star, $\mathcal{M}_{\mathrm{h}, \mathrm{c}}\left[M_{\odot}\right], \mathcal{R}_{\mathrm{h}, \mathrm{c}}\left[R_{\odot}\right]$, - stellar masses and mean radii of stars in solar units, $\log g_{\mathrm{h}, \mathrm{c}}-\log$ arithm (base 10) of the system components effective gravity, $M_{\text {bol }}^{\mathrm{h}, \mathrm{c}}$ - absolute bolometric magnitudes of NN Vir components and $a_{\mathrm{orb}}\left[R_{\odot}\right]$ - orbital semi-major axis in units of solar radius. 
of observations $n$, the final sum of the squares of the residuals between observed (LCO) and synthetic (LCC) light curves

$\sum_{i=1}^{n}\left(\mathrm{O}_{i}-\mathrm{C}_{i}\right)^{2}$,

and the standard deviation of the residuals

$\sigma=\sqrt{\frac{\sum_{i=1}^{n}\left(\mathrm{O}_{i}-\mathrm{C}_{i}\right)^{2}}{(n-1)}}$.

In the same table the spot characteristics (spot temperature factor, $A_{\mathrm{S}}=T_{\mathrm{S}} / T_{\mathrm{c}}$, angular radius, $\theta_{\mathrm{S}}$ and longitude, $\lambda_{\mathrm{S}}$ ) are also given. The determination of these parameters is based on a simultaneous fitting of the available light curves in the $B$ and $V$ photometric bands.

Finally, in Table 4 we present some important absolute parameters of the system. They are derived from the mass ratio of the components $q=m_{\mathrm{c}} / m_{\mathrm{h}}$, estimated by Rucinski \& Lu (1999) from radial velocity solution.

From the solutions obtained we can see that the secondary is the cooler component of the system, and that the mean gravity of the secondary is somewhat smaller than that of the primary, which holds well for A-type W UMa systems.

The estimated errors of the parameters arise from the nonlinear least-squares method, on which the inverse-problem method is based. The uncertainties of these parameters may be larger than we estimated. Our estimate of the accuracy in the determination of these parameters is based on the influence of formal errors arising from the nonlinear method of the light-curve analysis. Keeping in mind the errors of the input parameters of the model, which are treated as fixed in the inverse-problem method, the real errors of the parameters will definitely be larger (approximately 2-3 times).

Using the inverse-problem solutions for individual light curves, Fig. 2 gives a graphic presentation of these results, where the optimum fit of the observed light curves (LCO) to the synthetic light curves (LCC) is shown. The $\mathrm{O}-\mathrm{C}$ residuals between the observed (LCO) and optimum synthetic (LCC) light curves are also given. This figure shows the view of the Roche model of the system NN Vir, obtained with the parameters estimated by analysing the light curves. Using such plots, one can see how the system would seem at a certain orbital phase. Finally, at the bottom of the figure we show the $B$ and $V$ observations of the system, and the corresponding $B-V$ colour curve. The phase dependence of the colour curve can be a consequence of the existing hot region in the neck zone on the cooler secondary component.

It is evident in Table 4 and Fig. 2 that the Roche model with a relatively large hot area on the less massive star, near the neck region between the components gives a satisfactory fit to the analysed light curves. Here, in this region the temperature is increased by $23 \%$ compared to the surrounding photosphere, which is a consequence of the intensive energy transfer from the primary to the secondary through the neck region.

In the analysis of these light curves, the inclination of the orbit was estimated to be $i \sim 63^{\circ} 4$, which suggests partial eclipses in both of the light curve minima. The filling coefficient for the critical Roche lobe $F_{\mathrm{h}} \sim 1.074$ indicates a distinct overcontact configuration with a high degree of overcontact $f_{\text {over }} \sim 58 \%$.

\section{Conclusions}

A summary of our results, given in Table 4, proves that a Roche model with a hot active region on the less massive component of NN Vir can succesfully simulate the observed light curves. Synthetic light curves, obtained by solving the inverse problem, fit the observations very well, and we have quite good agreement between the solutions for individual light curves in different $B V$ filters of the photometric system. All this suggests the suitability of our Roche model with the hot area on the less massive star in simulating the real observations. Without this active region we have a fit of a much poorer quality.

The results describe the NN Vir system as a high overcontact configuration $\left(f_{\text {over }} \sim 58 \%\right)$ with a relatively small temperature difference between the components $\left(\Delta T=T_{\mathrm{h}}-T_{\mathrm{c}} \sim\right.$ $160 \mathrm{~K})$. They also suggest a significant mass and energy transfer from the more massive primary onto the less massive secondary. The hot area on the less massive star, near the neck region, is a consequence of this mass and energy exchange.

Acknowledgements. This work has been supported by the Ministry for Sciences and Technology of Serbia through the project 1191 "Stellar physics" and by the Turkish Academy of Sciences, in the framework of the Young Scientist Award Program (BA/TÜBA-GEBIP/2001-2-2). This research has made use of the Simbad database, operated at CDS, Strasbourg, France. We appreciate the anonymous referee for her/his constructive and generous comments.

\section{References}

Albayrak, B., Tanrıverdi, T., \& Aydın, C. 2002, IBVS, No. 5300

Carbon, D., \& Gingerich, O. 1969, in Theory and Observation of Normal Stellar Atmospheres, ed. O. Gingerich (Cambridge: MIT Press), 377

Claret, A. 1998, A\&AS, 131, 395

Claret, A. 2000, A\&A, 363, 1081

Djurašević, G. 1992a, Ap\&SS, 196, 241

Djurašević, G. 1992b, Ap\&SS, 197, 17

Djurašević, G., Zakirov, M., Hojaev, A., \& Arzumanyants, G. 1998, A\&AS, 131, 17

ESA 1997, The Hipparcos and Tycho Catalogs, SP-1200

Gomez-Forrelland, J. M., \& Garcia-Melendo, E. 1997, IBVS, No. 4469

Lejeune, T., Cuisinier, F., \& Buser, R. 1997, A\&AS, 125, 229

Lejeune, T., Cuisinier, F., \& Buser, R. 1998, A\&AS, 130, 65

Kwee, K. K., \& van Woerden, H. 1956, Bull. Astr. Inst. Neth., 12, 327

Lucy, L. B. 1967, Zs. f. Ap., 65, 89

Lucy, L. B., \& Wilson, R. E. 1979, ApJ, 231, 502

Marquardt, D. W. 1963, J. Soc. Ind. Appl. Math., 11(2), 431

Müyesseroğlu, Z. 2003, private communication

Popper, D. M. 1980, ARA\&A, 18, 115

Press, W. H., Teukolsky, S. A., Vetterling, W. T., \& Flannery, B. P. 1992, Numerical Recipes in Fortran, The Art of Scientific Computing, Second Edition (Cambridge: University Press, New York), 120

Rafert, J. B., \& Twigg, L. W. 1980, MNRAS, 139, 78

Rucinski, S. M. 1969, Acta Astr., 19, 245

Rucinski, S. M., \& Lu, W. 1999, AJ, 118, 2451

Wilson, R. E., \& Devinney, E. J. 1971, ApJ, 166, 605

Woitas, J. 1997, IBVS, No. 4444 